\section{Original Article}

Check for updates

\section{OPEN ACCESS}

Received: May 18, 2018

Revised: Jul 25, 2018

Accepted: Aug 7, 2018

Correspondence to

Young-Min Ye, MD, PhD

Department of Allergy and Clinical

Immunology, Ajou University School of

Medicine, 206 World cup-ro, Yeongtong-gu,

Suwon 16499, Korea.

Tel: +82-31-219-4262

Fax: +82-31-219-4265

E-mail: ye9007@ajou.ac.kr

Copyright (c) 2019 The Korean Academy of Asthma, Allergy and Clinical Immunology . The Korean Academy of Pediatric Allergy and Respiratory Disease

This is an Open Access article distributed under the terms of the Creative Commons Attribution Non-Commercial License (https:// creativecommons.org/licenses/by-nc/4.0/) which permits unrestricted non-commercial use, distribution, and reproduction in any medium, provided the original work is properly cited.

ORCID iDs

Ga-Young Ban (iD

https://orcid.org/0000-0002-7961-742X

Yoo Seob Shin iD

https://orcid.org/0000-0002-9855-3185 Hae-Sim Park iD

https://orcid.org/0000-0003-2614-0303

Young-Min Ye iD

https://orcid.org/0000-0002-7517-1715

Disclosure

There are no financial or other issues that might lead to conflict of interest.

\title{
Adaptation and Validation of the Korean Version of the Urticaria Control Test and Its Correlation With Salivary Cortisone
}

\author{
Ji-Ho Lee, ${ }^{1}$ Yoon Ju Bae, ${ }^{2}$ So-Hee Lee, ${ }^{3}$ Su-Chin Kim, ${ }^{4}$ Hyun-Young Lee, ${ }^{4}$ \\ Ga-Young Ban $(1),{ }^{5}$ Yoo Seob Shin $\left(\mathbb{1 0},{ }^{3}\right.$ Hae-Sim Park $\left(\mathbb{1},{ }^{3}\right.$ Juergen Kratzsch, ${ }^{2}$ \\ Young-Min $\mathrm{Ye} \mathbb{1 0}^{3^{*}}$
}

'Department of Internal Medicine, Yonsei University Wonju College of Medicine, Wonju, Korea ${ }^{2}$ Institute of Laboratory Medicine, Clinical Chemistry and Molecular Diagnostics, University of Leipzig, Leipzig, Germany

${ }^{3}$ Department of Allergy and Clinical Immunology, Ajou University School of Medicine, Suwon, Korea ${ }^{4}$ Clinical Trial Center, Ajou University Medical Center, Suwon, Korea

${ }^{5}$ Department of Pulmonary, Allergy and Critical Care Medicine, Kangdong Sacred Heart Hospital, Hallym University College of Medicine, Seoul, Korea

\section{ABSTRACT}

Purpose: Frequent changes in chronic urticaria (CU) activity over time can cause psychological stress, which also serves as a trigger of CU. To measure the control status of CU, the Urticaria Control Test (UCT) was developed in Germany. This study aimed to investigate the validity, reliability and responsiveness to changes in CU for the Korean version of the UCT (K-UCT) and its relation with salivary cortisol and cortisone levels.

Methods: Linguistic adaptation of the UCT into Korean was conducted. A total of $96 \mathrm{CU}$ patients were enrolled, and 80 of them completed the study. The K-UCT and other outcome scores for CU were measured and repeated after 4 weeks of treatment. Control status was classified by physicians into well-controlled, partly-controlled, and uncontrolled CU. Salivary cortisol and cortisone were measured by liquid chromatography-tandem mass spectrometry. Results: Excellent internal consistency and intra-class reliability were obtained. Strong correlations between the K-UCT and disease severity, reflected in the Urticaria Activity Score (UAS)/global assessment of urticaria control by physicians/patient assessment of symptom severity/CU-specific quality of life were noted. K-UCT scores $\geq 12$ were found to be optimal for determining well-controlled CU (sensitivity, $75.0 \%$; specificity, $758 \%$; area under the curve, 0.824). Perceived stress scale scores were significantly correlated with the UAS and the K-UCT. Salivary cortisone levels were significantly correlated with K-UCT $(r=0.308, P=0.009)$ and differed significantly according to control status determined by a K-UCT $\geq 12$.

Conclusions: This study demonstrated that the K-UCT can be a valid instrument with which to gauge $\mathrm{CU}$ control status in Korean patients. Further studies are needed to validate salivary cortisone as a biomarker for $\mathrm{CU}$ control.

Keywords: Chronic urticaria; urticaria control test; linguistic adaptation; validation; Korea 


\section{INTRODUCTION}

Chronic urticaria (CU) is defined as the occurrence of wheals, angioedema or both for more than 6 weeks. ${ }^{1} \mathrm{CU}$ is not life-threatening, although its psychological impact has been found to be comparable to that of coronary artery disease. ${ }^{2}$ The lifetime prevalence of $\mathrm{CU}$ is estimated to range from $0.05 \%$ to $3 \% .^{3}$ A nationwide epidemiologic study performed in Korea using health insurance data revealed remarkable increases in the prevalence of CU between 2010 and $2014 .^{4}$

Patients with CU are usually treated following standardized guidelines in which treatment steps are adjusted according to symptom severity and responses to prior therapies. ${ }^{1}$ The clinical manifestation of CU fluctuates in its severity, with a considerable number of patients presenting in an allergy clinic without any symptoms and objective signs. Therefore, evaluating their treatment response can be a challenge. The Urticaria Activity Score (UAS) and Chronic Urticaria Quality of Life Questionnaire (CU-Q2oL) have been recommended as measures to assess disease activity and its impact on quality of life. ${ }^{1}$ However, the treatment goal of CU is complete symptom control, which cannot be assessed by the UAS and CUQ2oL. Therefore, the Urticaria Control Test (UCT) was invented as an effort to address the lack of tools for assessing disease control. ${ }^{5}$

CU has been found to be associated with chronic stress, ${ }^{6}$ which can, in turn, affect CU activity. Stimulation of the hypothalamus-pituitary-adrenal (HPA) axis by stress leads to cortisol secretion from the adrenal cortex. Since more than $90 \%$ of cortisol in circulation binds to the corticosteroid-binding globulin, salivary cortisol reflects the biologically active form of serumfree cortisol. ${ }^{8}$ Since the salivary glands exhibit high levels of $11 \beta$-hydroxysteroid dehydrogenase $2,{ }^{9}$ salivary cortisol is rapidly converted to cortisone resulting in a 2-6 times higher concentration of cortisone than cortisol in saliva. ${ }^{10}$ Meanwhile, recent technology has enabled researchers to correctly measure cortisol and cortisone levels from saliva. Indeed, increasing evidence indicates that salivary cortisol and cortisone levels mirror those in the blood. ${ }^{11}$

In the present study, we aimed to translate the original UCT into Korean (K-UCT) and to investigate its validity, reliability, applicability and associations with subjective stress scores and salivary cortisol and cortisone levels.

\section{MATERIALS AND METHODS}

\section{Study population}

Korean patients with $\mathrm{CU}$ aged 19 years or older were recruited. All patients could read and write the Korean language and provided signed informed consent. Patients with chronic dermatological diseases other than $\mathrm{CU}$, those with other chronic disease associated with pruritus and those with diseases of the hypothalamic-pituitary-adrenal axis, such as the central nervous system and endocrine system, or other chronic inflammatory and autoimmune conditions that can affect serum cortisol levels were excluded. This study was approved by the Institutional Review Board of Ajou University Hospital (AJIRB-MED-OBS-14-294).

On the first visit (visit 1), demographics, past disease history, drug use, smoking history, and detailed information on the diagnosis of $\mathrm{CU}$ were obtained from the patients. The presence of atopy was determined by a skin prick test or specific IgE for common inhalant allergens. An 
autologous serum skin test was performed following the method suggested by the European Academy of Allergy and Clinical Immunology (EAACI)/Global Allergy and Asthma European Network (GA $\left.{ }^{2} L E N\right)$ task force. ${ }^{12}$ Positivity was determined when wheals induced by autologous serum reached sizes at least $1.5 \mathrm{~mm}$ greater than those induced by a negative control.

Standardized CU treatment was given to patients following EAACI/GA²LEN/European Dermatology Forum (EDF)/World Allergy Organization (WAO) guidelines. ${ }^{1}$ Treatment steps were classified according to the number of antihistamines and leukotriene receptor antagonists, cyclosporine or systemic corticosteroids. Patient-oriented questionnaires to assess urticaria activity, CU-specific quality of life (CU-QoL), symptom severity, perceived stress, depression and K-UCT were completed by patients before and after 4 weeks of treatment (visit 2). Additionally, physicians evaluated the urticaria symptoms of the patients using the visual analogue scale (VAS). Patients were instructed to use an internet website to complete the UAS on their own and to record UAS results on the website each day durig the study period.

\section{Linguistic adaptation}

The original version of the UCT in German was respectively translated into Korean by 2 professional German translators for whom Korean is their native language. The respective translations were compared and put together through discussion between researchers and allergy specialists. Backward translation into German was conducted by a professional Korean translator whose native language is German. This was then harmonized with the original UCT through examination of the translation process and the results as a way of reducing discrepancies and errors. The final version of the Korean UCT was tested in 5 patients with CU who showed no problems in interpreting, understanding and utilizing the questionnaires. The final version of the K-UCT was applied in this study (Supplementary Fig. S1).

\section{Patient-reported outcome measures for CU}

Various scales were employed to measure CU-related clinical features. The original UAS consists of 2 items, number of wheals and pruritus severity. Three items, distribution, duration and diameter of wheals, were added in the UAS-Ye (UAS revised by Ye et al.). ${ }^{14}$ Each item is scored between 0 and 3 for a maximum total value of 15 , which indicates most actively distressing urticaria. Urticaria Activity Score Over 7 Days (UAS7) is a sum of the original 2-item UAS for a consecutive 7 days (range, 0-42). ${ }^{13}$ The CU-QoL questionnaire developed and validated for measuring quality of life (QoL) in Korean CU patients was also administered. ${ }^{14}$ CU-QoL comprises 17 items (0-4 points, respectively). Total scores are transformed to a scale ranging from 0 to 100, with higher scores indicating better QoL. The VAS was assessed by patients and physicians independently to measure the severity of CU from different perspectives. Higher scores in patient VAS severity indicate more severe CU (range, 0-100), while higher scores in physician-derived VAS control reflect more controlled CU (range, 0-100). In addition, psychologic stress was assessed using the Perceived Stress Scale (PSS), which comprises 14 items (0-4 points, respectively), with total scores ranging from 0 to 56 . Depression was measured using the Center for Epidemiologic Studies-Depression (CESD) scale, which comprises 10 items (1-4 points, respectively), with total scores ranging from 10 to 40. Both PSS and CESD have been validated in the Korean population, with higher scores indicating more severe degrees of stress and depression. ${ }^{15}$

\section{Determination of urticaria control}

Physicians determined and classified the status of CU patients into 3 categories at each visit: well-controlled, partly-controlled and uncontrolled. Responsiveness to treatment 
was assessed at the second visit. A favorable responder was defined as maintaining wellcontrolled status or having improved urticaria control, such as from partly-controlled to well-controlled and from uncontrolled to partly- or well-controlled, after the 4-week treatment for CU. A poor responder was defined as staying continuously at partly-controlled or uncontrolled status or having deteriorated urticaria control.

\section{Validation of K-UCT}

Known-groups validity of the UCT was analyzed in regard to whether the K-UCT was discriminative according to physician-assessed urticaria control, between well- and partlycontrolled states with uncontrolled status. In addition, changes in K-UCT were compared between control statuses and responders at the second visit. The internal consistency of the 4 items in the K-UCT was measured using the Cronbach $\alpha$ coefficient. Cronbach $\alpha$ was interpreted as follows: $<0.60$, unacceptable; $0.60-0.65$, undesirable; $0.65-0.70$, minimally acceptable; 0.70-0.80, respectable; 0.80-0.90, excellent; and >0.90, excessive consistency. ${ }^{5}$ The intra-class correlation coefficient between physician VAS control and K-UCT was also measured. Therein, coefficient values were interpreted as follows: $<0.40$, poor; 0.40-0.60, fair; 0.60-0.75, good; and 0.75-1.00, excellent. ${ }^{16}$

Convergent validity was assessed in regard to whether the K-UCT is correlated with other CU associated clinical scores (i.e., UAS-Ye, UAS7, CU-QoL, physician VAS control, patients VAS severity, PSS and CESD). Responsiveness of the K-UCT in accordance with treatment for CU patients was estimated as 2 different cutoff values: First, the specific value at which wellcontrolled CU was optimally discriminated from partly-controlled or uncontrolled CU was analyzed. Secondly, the minimal important difference (MID) was estimated, which means the minimal change in the K-UCT score with which to differentiate favorable responders from poor responders.

\section{Measurement of salivary cortisol and cortisone levels}

Saliva samples were collected using Salivette ${ }^{\circledR}$ tubes for cortisol (Sarstedt, Sevelen, Switzerland) at the outpatient visit in the morning ( 9 to $11 \mathrm{AM}$ ). Eating and brushing teeth were restricted for 30 minutes before sampling. Collected saliva was centrifuged and aliquoted for measuring cortisol and cortisone concentrations as well as alpha-amylase activity. Samples were stored at $-80^{\circ} \mathrm{C}$ until ready for experimentation. Salivary cortisol and cortisone levels were analyzed by liquid chromatography-tandem mass spectrometry as previously described. ${ }^{17}$ Salivary alpha-amylase was determined by an enzymatic calorimetric test using the Hitachi Cobas c System (Roche, Manheim, Germany) according to the specifications and protocols provided by the manufacturer. ${ }^{10}$

\section{Statistical analysis}

Clinical characteristics were analyzed using $t$ tests for continuous variables and Pearson's $\chi^{2}$ test for categorical variables. Mean differences in the K-UCT were compared using independent $t$ tests between patients of different control status and responders. In convergent validity, a generalized estimating equations model was applied to evaluate correlations between the K-UCT and other CU associated scores adjusting for age, sex and treatment step. Receiver operating characteristics (ROC) and area under the curve (AUC) were used to evaluate screening accuracy and sensitivity of K-UCT in determining changes in urticaria control after treatment. Each score change in the K-UCT was analyzed to determine MID. Optimal change in the K-UCT to discriminate favorable and poor responders was determined by considering sensitivity and specificity obtained from AUC. 
Correlations between salivary cortisol and cortisone levels and CU related scores, PSS, and CESD were analyzed by Pearson's correlation coefficient $(r)$. A $P$ value less than 0.05 was considered statistically significant. All statistical analyses were performed using SPSS version 23.0 (IBM Corp., Armonk, NY, USA). To explore the associations between K-UCT in relation to UAS7, CU-QoL, physician VAS control, and salivary cortisone, path analysis using Amos 21.0 software (Small Waters Corp., Chicago, IL, USA) was performed. Structural equation modeling was used to evaluate the fit of the final model by examining a number of statistics. To examine the magnitude of the discrepancy between the sample and fitted covariance matrices, the $\chi^{2}$ test was used when a nonsignificant test indicated that the model and data were consistent..$^{18,19}$ The comparative fit index examines the difference in overall fit among the models, and values $>0.95$ indicate a good model fit. The root mean square error of approximation evaluates the approximate fit of the model, with values $<0.005$ suggesting a reasonable goodness of fit. ${ }^{18,19}$

\section{RESULTS}

\section{Study subjects}

Of the 96 patients with CU who were eligible for this study, 80 completed the whole study process. The mean age was $37.9 \pm 11.7$ years, and $52(65.0 \%)$ patients were female. Salivary cortisol and cortisone levels, as well as treatment steps, were comparable between visits 1 and 2. The K-UCT, UAS, CU-QoL, physician VAS control, patients VAS severity, and PSS scores were significantly improved at visit 2 compared t visit 1 (Table 1). The proportion of patients with

Table 1. Clinical characteristics of the study subjects

\begin{tabular}{|c|c|c|c|}
\hline Characteristics & Visit $1(n=96)$ & Visit $2(n=80)$ & $P$ value* \\
\hline Age (yr) & $38.1 \pm 11.8$ & $37.9 \pm 11.7$ & \\
\hline Female $(\%)$ & $62(64.6)$ & $52(65.0)$ & \\
\hline Atopy (\%) & $48(50.0)$ & $41(51.3)$ & \\
\hline Total IgE (kU/L) & $234.3 \pm 279.1$ & $234.2 \pm 289.8$ & \\
\hline Angioedema (\%) & $42(43.7)$ & $37(46.3)$ & \\
\hline UAS-Ye (0-15) & $7.1 \pm 3.6$ & $5.0 \pm 3.5$ & $<0.001$ \\
\hline UAS7 (0-42) & $10.4 \pm 8.4$ & $9.1 \pm 8.3$ & 0.061 \\
\hline CU-QoL (0-100) & $64.7 \pm 20.9$ & $80.5 \pm 18.9$ & $<0.001$ \\
\hline K-UCT (0-16) & $8.4 \pm 3.6$ & $11.5 \pm 3.1$ & $<0.001$ \\
\hline Physician VAS control (0-100) & $55.6 \pm 25.1$ & $76.1 \pm 26.0$ & $<0.001$ \\
\hline Patients VAS severity (0-100) & $41.7 \pm 28.8$ & $24.0 \pm 24.2$ & $<0.001$ \\
\hline PSS (0-56) & $23.2 \pm 6.9$ & $20.2 \pm 6.9$ & $<0.001$ \\
\hline CESD (10-40) & $17.6 \pm 4.8$ & $16.5 \pm 4.8$ & 0.284 \\
\hline Salivary cortisone (nmol/L) & $27.2 \pm 16.7$ & $21.5 \pm 12.1$ & 0.105 \\
\hline Salivary cortisol (nmol/L) & $3.5 \pm 3.7$ & $3.8 \pm 9.1$ & 0.986 \\
\hline Salivary $\alpha$-Amylase (uKat/L) & $9.6 \pm 8.8$ & $6.9 \pm 8.9$ & 0.355 \\
\hline Serum IL-6 (pg/mL) & $3.5 \pm 10.2$ & $10.1 \pm 70.3$ & 0.396 \\
\hline Treatment step (\%) & & & 0.100 \\
\hline No medication & $7(7.3)$ & $0(0.0)$ & \\
\hline$\leq 2$ antihistamines & $40(41.7)$ & $33(41.3)$ & \\
\hline 3-4 antihistamines & $17(17.7)$ & $16(20.0)$ & \\
\hline With LTRA & $10(10.4)$ & $13(16.3)$ & \\
\hline With cyclosporine \pm steroid & $22(22.9)$ & $18(22.5)$ & \\
\hline Control status (\%) & & & $<0.001$ \\
\hline Well & $15(15.6)$ & $33(41.3)$ & \\
\hline Partly & $56(58.3)$ & $40(50.0)$ & \\
\hline Uncontrolled & $25(26.0)$ & $7(8.8)$ & \\
\hline
\end{tabular}

Values are presented as number (\%) or mean \pm standard deviation.

UAS-Ye, Urticaria Activity Score revised by Ye et al.; UAS7, Urticaria Activity Score Over 7 Days; CU-QoL, chronic urticaria-specific quality of life; K-UCT, Korean version of the Urticaria Control Test; VAS, visual analogue scale; PSS, Perceived Stress Scale; CESD, Center for Epidemiologic Studies-Depression; LTRA, leukotriene receptor antagonist.

${ }^{*}$ Paired $t$ test 


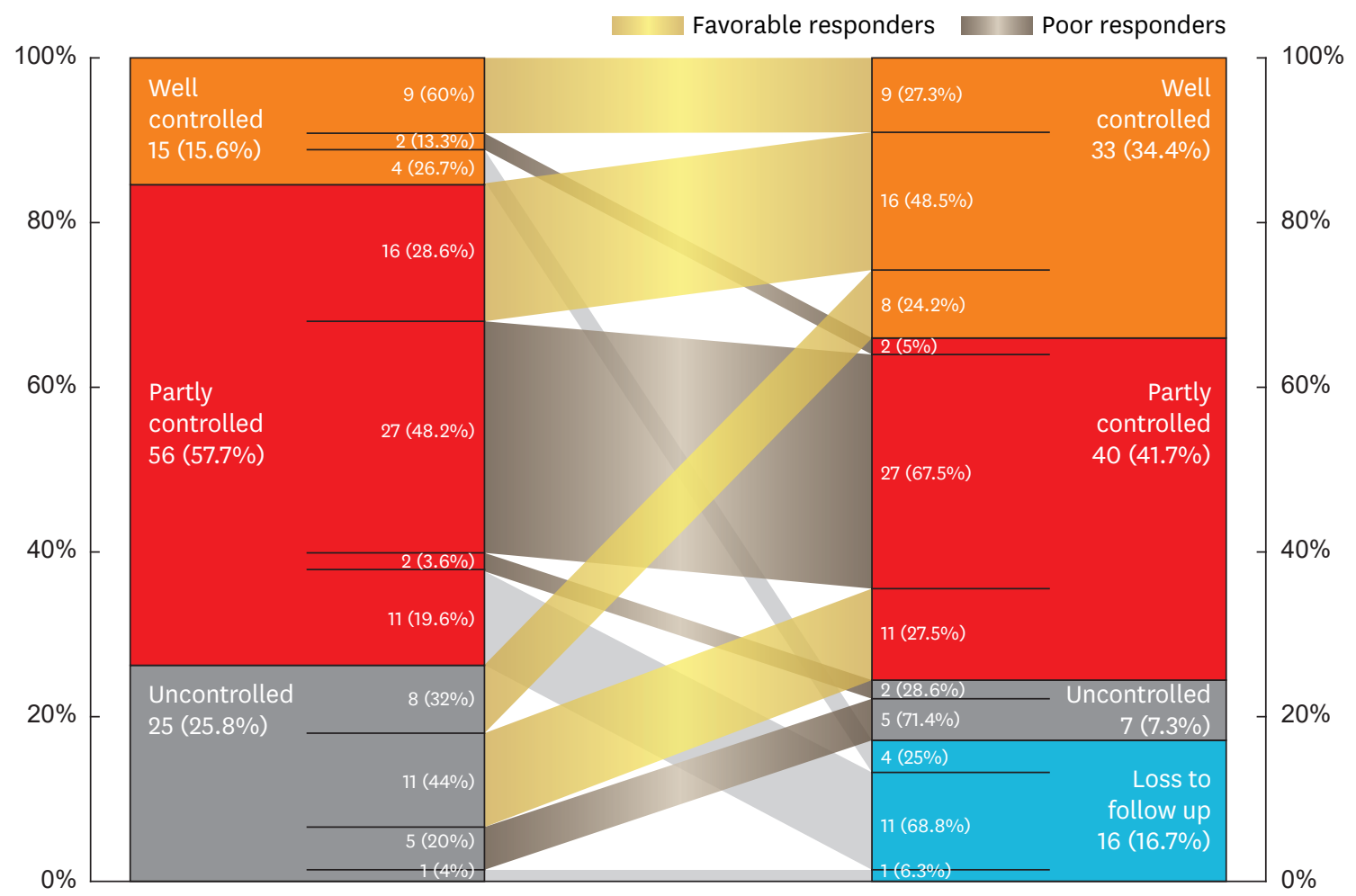

Fig. 1. Changes in patient distributions according to control status after 4 weeks of treatment.

well-controlled CU increased from $13.8 \%$ to $41.2 \%$, and those with uncontrolled CU decreased from $30.0 \%$ to $8.8 \%$ after 4 weeks of treatment $(P<0.001$, Fig. 1$)$. The 80 patients who completed the study were classified into favorable $(n=44)$ and poor $(n=36)$ responders. In the favorable responder group, 9 patients maintained well-controlled CU; 16 patients moved from partly-controlled CU to well-controlled CU; and 8 and 11 patients with uncontrolled CU moved to well- and partly-controlled CU, respectively. The poor responder group comprised 2 patients who moved from well-controlled CU to partly-controlled CU, 27 patients who maintained partly-controlled status, 2 patients who moved from partly-to un-controlled $\mathrm{CU}$, and 5 patients who maintained uncontrolled CU (Fig. 1).

\section{Validation of K-UCT}

The 4 items of the K-UCT were found to have excellent internal consistency with a Cronbach $\alpha$ of 0.87 .The correlation between physician VAS control and K-UCT was also excellent, with an intra-class correlation coefficient of 0.77 .

After 4-week treatment, patients classified as exhibiting well-controlled CU showed significantly lower scores for the UAS, patients VAS severity, and PSS and higher scores for the CU-QoL, physician VAS control, and K-UC, compared to those with partly- or uncontrolled CU (Table 2). However, CESD and salivary cortisol and cortisone levels after treatment were not different between patients with well-controlled CU and partly- or uncontrolled CU.

In terms of responsiveness to treatment, increases in the K-UCT were significantly higher in favorable responders than in poor responders $(4.9 \pm 3.4$ vs. $1.6 \pm 2.5, P<0.001)$. Moreover, changes in UAS-Ye $(-3.5 \pm 3.0$ vs. $-1.0 \pm 2.9, P<0.001)$, CU-QoL ( $20.6 \pm 15.4$ vs. $12.8 \pm 13.6$, 
Table 2. Comparison of clinical characteristics according to the control status of CU assessed by physicians

\begin{tabular}{|c|c|c|c|}
\hline Characteristics & Well-controlled CU $(n=33)$ & Partly- or uncontrolled CU $(n=47)$ & $P$ value \\
\hline Age (yr) & $35.8 \pm 11.3$ & $39.3 \pm 11.9$ & 0.186 \\
\hline Female (\%) & $21(63.6)$ & 31 (66.0) & 0.830 \\
\hline Atopy (\%) & $17 / 31(54.8)$ & $24 / 45(53.3)$ & 0.897 \\
\hline Total IgE (kU/L) & $242.59 \pm 296.56$ & $228.45 \pm 288.22$ & 0.833 \\
\hline Angioedema (\%) & $8 / 24(33.3)$ & 6/32 (18.8) & 0.212 \\
\hline UAS-Ye (0-15) & $2.4 \pm 2.9$ & $6.8 \pm 2.7$ & $<0.001$ \\
\hline UAS7 (0-42) & $4.3 \pm 6.9$ & $12.7 \pm 7.4$ & $<0.001$ \\
\hline CU-QoL (0-100) & $90.0 \pm 12.5$ & $73.8 \pm 19.9$ & $<0.001$ \\
\hline K-UCT (0-16) & $13.5 \pm 2.3$ & $10.2 \pm 2.9$ & $<0.001$ \\
\hline Physician VAS control (0-100) & $96.9 \pm 4.9$ & $61.4 \pm 24.8$ & $<0.001$ \\
\hline Patients VAS severity (0-100) & $12.3 \pm 19.0$ & $32.3 \pm 24.3$ & $<0.001$ \\
\hline PSS (0-56) & $17.2 \pm 7.8$ & $22.2 \pm 5.3$ & 0.002 \\
\hline CESD (10-40) & $16.0 \pm 5.0$ & $16.8 \pm 4.7$ & 0.458 \\
\hline Salivary cortisone (nmol/L) & $28.0 \pm 21.6$ & $24.8 \pm 13.5$ & 0.481 \\
\hline Salivary cortisol (nmol/L) & $4.1 \pm 5.5$ & $3.0 \pm 2.3$ & 0.271 \\
\hline Treatment step (\%) & & & 0.211 \\
\hline No medication & $4(12.1)$ & $3(6.4)$ & \\
\hline$\leq 2$ antihistamines & $16(48.5)$ & $15(31.9)$ & \\
\hline 3-4 antihistamines & $6(18.2)$ & $7(14.9)$ & \\
\hline With LTRA & $2(6.1)$ & $8(17.1)$ & \\
\hline With cyclosporine and/or steroid & $5(15.2)$ & $14(29.8)$ & \\
\hline
\end{tabular}

All values givens were measured at visit 1 and mean \pm standard deviation.

CU, chronic urticaria; UAS-Ye, Urticaria Activity Score revised by Ye et al.; UAS7, Urticaria Activity Score Over 7 Days; CU-QoL, chronic urticaria-specific quality of life; K-UCT, Korean version of Urticaria Control Test; VAS, visual analogue scale; PSS, Perceived Stress Scale; CESD, Center for Epidemiologic Studies-Depression; LTRA, leukotriene receptor antagonist.

$P=0.021)$, and Physician VAS $(32.3 \pm 27.9$ vs. $6.1 \pm 24.6, P<0.001)$ were also significantly different between favorable and poor responders. However, changes in UAS7 ( $-2.2 \pm 5.8$ vs. $-0.1 \pm 6.0, P=0.126)$, patient VAS $(-21.6 \pm 30.6$ vs. $-16.6 \pm 21.0, P=0.409)$, PSS $(-3.8 \pm 6.7$ vs. $-1.6 \pm 5.5, P=0.120)$, and CESD $(-0.7 \pm 5.7$ vs. $-0.4 \pm 3.7, P=0.814)$ were not significantly different between the 2 response groups (Fig. 2).

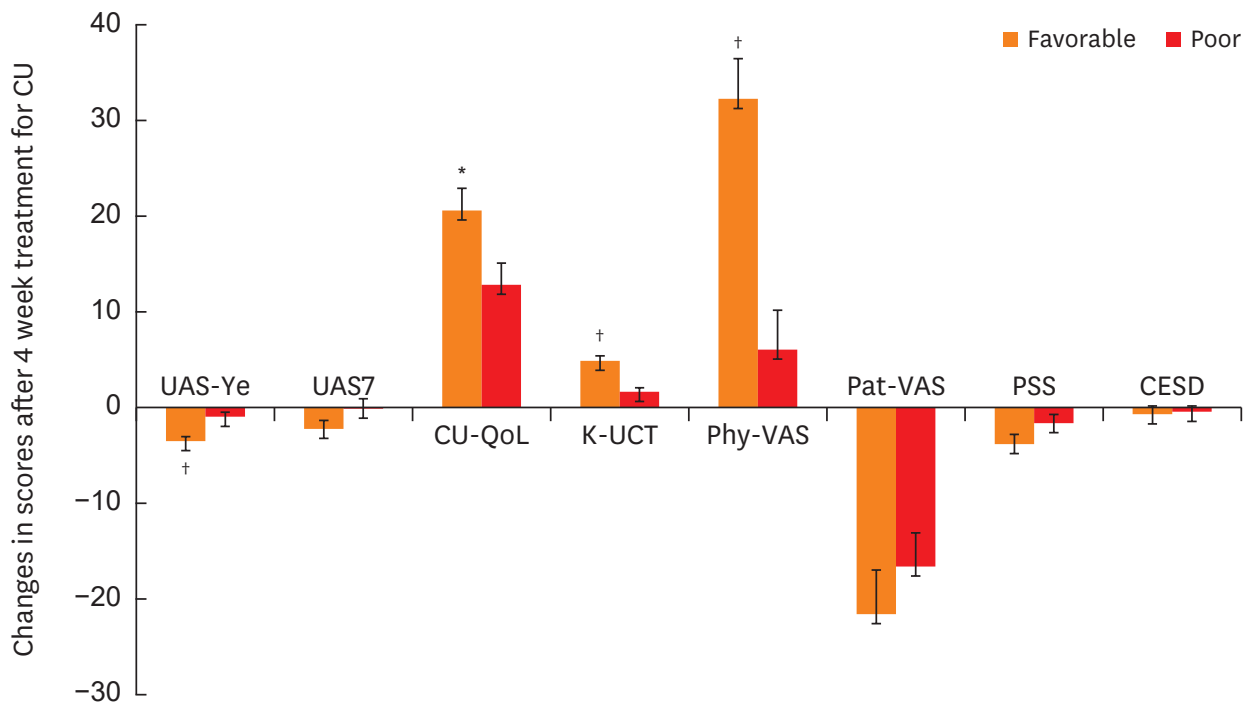

Fig. 2. Changes in CU related scores after 4-week treatment. Positive values mean increasing scores at visit 2 . On the contrary, negative scores represent decreasing scores at visit 2.

CU, chronic urticaria; UAS-Ye, Urticaria Activity Score revised by Ye et al.; UAS7, Urticaria Activity Score Over 7 Days; CU-QoL, chronic urticaria-specific quality of life; K-UCT, Korean version of Urticaria Control Test; VAS, visual analogue scale; PSS, Perceived Stress Scale; CESD, Center for Epidemiologic Studies-Depression.

${ }^{*} P<0.05,{ }^{\dagger} P<0.001$. 
Table 3. Correlation analyses between the K-UCT and other outcome measures

\begin{tabular}{|c|c|c|c|c|}
\hline \multirow[t]{2}{*}{ Measurements } & \multirow[t]{2}{*}{$P$ value } & \multirow[t]{2}{*}{$\operatorname{Exp}(\beta)$} & \multicolumn{2}{|c|}{$95 \% \mathrm{Cl}$} \\
\hline & & & Lower & Upper \\
\hline UAS-Ye (0-15) & $<0.001$ & 0.541 & 0.468 & 0.624 \\
\hline UAS7 (0-42) & $<0.001$ & 0.320 & 0.230 & 0.445 \\
\hline CU-QoL (0-100) & $<0.001$ & 53.644 & 25.697 & 111.987 \\
\hline Physician VAS control (0-100) & $<0.001$ & 101.804 & 46.700 & 219.929 \\
\hline Patients VAS severity (0-100) & $<0.001$ & 0.008 & 0.003 & 0.020 \\
\hline PSS (0-56) & $<0.001$ & 0.467 & 0.339 & 0.643 \\
\hline CESD (10-40) & 0.002 & 0.687 & 0.544 & 0.866 \\
\hline Salivary cortisol & 0.124 & 1.302 & 0.930 & 1.823 \\
\hline Salivary cortisone & 0.267 & 1.581 & 0.705 & 3.545 \\
\hline
\end{tabular}

A generalized estimating equations model adjusted for age, sex, and treatment step was applied. All values of visit 1 and visit 2 were included separately. K-UCT, Korean version of the Urticaria Control Test; CI, confidence interval; UAS-Ye, Urticaria Activity Score revised by Ye et al.; UAS7, Urticaria Activity Score Over 7 Days; CU-QoL, chronic urticaria-specific quality of life; VAS, visual analogue scale; PSS, Perceived Stress Scale; CESD, Center for Epidemiologic Studies-Depression.

To determine convergent validity, a generalized estimating equations model adjusted for age, sex and treatment step was applied. The K-UCT was significantly dependent on all scores for patient-reported outcome measures, including UAS-Ye, UAS 7, CU-QoL, patient VAS, PSS and CESD, in addition to physician VAS (Table 3).

A ROC curve analysis showed that a K-UCT score of 12 was the optimal cutoff value with which to differentiate well-controlled CU from partly- or uncontrolled $\mathrm{CU}$, with a sensitivity of $75.0 \%$, a specificity of $75.8 \%$, and an AUC of $0.824(P<0.001)$. When the minimal change in the K-UCT associated with favorable responders was assessed by ROC, a K-UCT score of 1 had the highest sensitivity of $90.9 \%$, but a low specificity of $36.1 \%$. Given that the mean increment in K-UCT scores in poor responders was 1.6, a K-UCT score of 2 was considered as the optimal cutoff value with which to detect favorable responders with a sensitivity of $81.8 \%$, a specificity of $50.0 \%$ and an AUC of $0.772(P<0.001)$.

\section{Salivary cortisol and cortisone}

Salivary cortisol and cortisone levels were well correlated each other $(r=0.839, P<0.001)$. PSS had a significant negative correlation with salivary cortisol $(r=-0.267, P=0.023)$ and cortisone $(r=-0.261, P=0.027)$. On the contrary, K-UCT showed a significant positive correlation with salivary cortisone $(r=0.308, P=0.009)$. Salivary cortisone levels were also significantly higher among patients with controlled CU showing a K-UCT score of $\geq 12$ than those with a K-UCT of $<12(3.55 \pm 4.39$ vs. $2.21 \pm 2.16 \mathrm{nmol} / \mathrm{L}, P=0.011)$.

\section{Structural equation modeling}

A structural equation model was developed to evaluate the relationships among K-UCT and UAS7, CU-QoL/Physician VAS for urticaria control/salivary cortisone levels (Fig. 3). To investigate the determinants of CU-QoL, the final outcome of CU, we considered that K-UCT and UAS7 by itself or through direct or indirect interactions with physician VAS for urticaria control could influence disease activity.

The goodness of fit of the model was demonstrated using several fit statistics. A $\chi^{2}$ test of absolute fit was not significant $(r=5.263, P=0.261)$, indicating that this model was a good fit for the data. This was further supported by a root mean square error of approximation value of 0.068 (90\% confidence interval [CI], 0.000-0.204) and a comparative fit index value $>0.95$. The model had a relatively small Akaike information criterion value (27.263), suggesting that this simple model may provide a good explanation of the relationships among K-UCT and UAS7, CU-QoL/physician VAS/ and salivary cortisone levels. The regression coefficient $\beta$ represents 


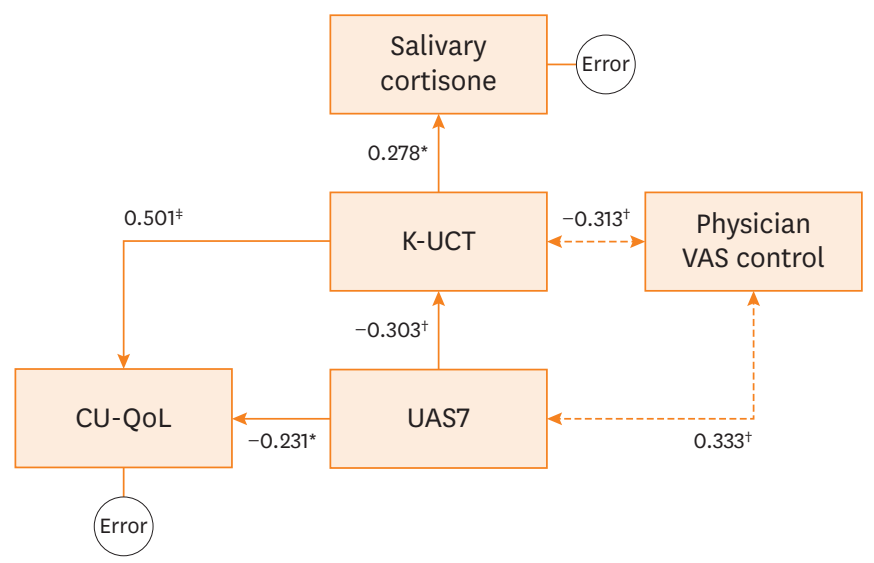

Fig. 3. Structural equation modeling to estimate relationships among K-UCT and UAS7/CU-QoL/salivary cortisone levels. The K-UCT was found to be a significant predictor of salivary cortisone levels $(\beta=0.278, P=0.016)$ and $\mathrm{CU}-\mathrm{QoL}$ $(\beta=0.501, P<0.001)$. The UAS7 negatively impacted both K-UCT $(\beta=-0.303, P=0.008)$ and CU-QoL $(\beta=-0.231$, $P=0.021$ ). The numbers on the arrows indicate the regression coefficient $(\beta)$ of the structural equation model. Positive numbers mean a positive correlation between 2 factors, whereas negative numbers mean negative correlations. The direction of the arrows represents cause and effect.

K-UCT, Korean version of Urticaria Control Test; CU, chronic urticaria; UAS7, Urticaria Activity Score Over 7 Days; CU-QoL, chronic urticaria-specific quality of life.

${ }^{*} P<0.05,{ }^{\dagger} P<0.01,{ }^{\ddagger} P<0.001$.

the index of path direction and the intensity of the relation between two factors. The K-UCT was found to be a significant predictor of CU-QoL $(\beta=0.501, P<0.001)$ and salivary cortisone levels $(\beta=0.278, P=0.016)$. A significant impact of UAS7 on CU-QoL $(\beta=-0.231, P=0.021)$ and K-UCT $(\beta=-0.303, P=0.008)$ was also identified in the model.

\section{DISCUSSION}

The Korean version of the UCT was successfully translated and validated in the present study. K-UCT scores were higher in well-controlled CU as determined by a physician than in partlyor uncontrolled CU, and increases in the K-UCT after 4 weeks of treatment were larger in favorable responders than in poor responders. Excellent internal consistency for the 4 items of the K-UCT and intra-class correlation between physician VAS control and K-UCT were identified. We also noted significant correlations of K-UCT with UAS-Ye, UAS 7, CU-QoL, physician and patient VAS, PSS and CESD. Additionally, we demonstrated that the optimal cutoff value of K-UCT scores with which to discriminate well-controlled CU was 12. The MID for detecting favorable responders was a K-UCT score of 2. Finally, salivary cortisone levels were found to be positively correlated with K-UCT.

Patient-reported outcomes (PROs) have been recommended for assessing disease severity and treatment responses and have been adopted as primary and secondary outcomes in clinical trials. ${ }^{20}$ Until now, only 2 PROs, CU-Q2oL and UAS, have been translated and validated internationally. However, these measures do not reflect symptom control, which is the goal of CU treatment. ${ }^{1}$ To address the lack of an assessment tool for evaluating urticaria control, the UCT was recently developed first in Germany and translated in American English. ${ }^{5}$ After that, a Thai version of the UCT was introduced and validated in Thai patients with CU. ${ }^{21}$ Also, while the UCT was also translated into Spanish, its clinical utility was not validated in Spanish patients with CU. ${ }^{22}$ As far as we know, the K-UCT is the fourth translated version of the original German UCT. Herein, we found the K-UCT to be a valid PRO. The 
K-UCT was higher in patients with well-controlled CU than those with partly- or uncontrolled $\mathrm{CU}$, and larger increases in K-UCT after treatment were noted for favorable responder than for poor responders. In addition, K-UCT was strongly correlated with other CU-related scores, and CU-QoL was determined by K-UCT in a structural equation model.

In the present study, a K-UCT score of 12 was found to be accurate for discriminating wellcontrolled CU from partly- or uncontrolled CU, which is in agreement with the German and Thai versions of the UCT., ${ }^{5,21}$ This similarity in results suggests the applicability and consistency of the use of the UCT in other countries. In the case of asthma, an Asthma Control Test score of 20 has been used as a standard score in determining its control status. Therefore, given that a UCT score of 12 has been found to have the same clinical meaning in different linguistic and cultural settings, it is possible for that to be accepted as a practical standard when assessing and treating $\mathrm{CU}$ patients.

Prior to this study, the MID of the UCT has been evaluated in 2 countries, Germany and Thailand. Both studies reached the conclusion that a difference of 3 points in UCT scores is sufficient to differentiate clinical responders. ${ }^{21,23}$ However, in our study, we found a difference of 2 points in the K-UCT was enough to differentiate responders. This might be attributable to the different anchors used in determining the presence of a response and different treatment methods among studies. In the 2 aforementioned studies, patients were asked to rank their control state on a 5-point scale (no control, little control, moderate control, good control and complete control) for themselves as an anchor to determine control state..$^{21,23}$ In this study, the presence of a response, either favorable or poor, was determined by a physician using 3 control states (well-controlled, partly-controlled and uncontrolled). In addition, while all of the patients were treated with omalizumab in the German study, patients treated with omalizumab were not enrolled in the present study. ${ }^{23}$ Omalizumab is a potent biological agent that can elicit dramatic increases in the control step. Therefore, different methods of analysis and treatment methods as well as a cultural difference, need to be taken into consideration when interpreting MID differences between studies.

Evidence indicates that $\mathrm{CU}$ is associated with chronic stress. ${ }^{24}$ Chronic stress has been defined as a specific ongoing environmental condition such as marital discord and caretaker burden, or as a stressor with enduring impact over at least 4 weeks. ${ }^{25} \mathrm{~A}$ recent study demonstrated that 26 of 45 (57.8\%) CU patients exhibit hypocortisolism (defined by baseline serum morning cortisol levels $<3 \mu \mathrm{g} / \mathrm{dL}$ and poststimulation values $<18 \mu \mathrm{g} / \mathrm{dL}$ ). Therein, interleukin 18 and high sensitivity C-reactive protein, which are typically regulated by cortisol, were found to be positively correlated with UAS, whereas cortisol was negatively correlated with UAS. ${ }^{26}$ This result indicated that chronic stress caused by CU decreases cortisol level and increases inflammatory mediators. The hypothalamic-pituitary-adrenal axis is activated by acute stress, while it is attenuated in response to chronic stress. ${ }^{27}$ Cortisol is converted to cortisone by $11 \beta$-hydroxysteroid dehydrogenase, which is augmented in salivary glands. ${ }^{11}$ Salivary cortisol and cortisone concentrations show strong correlations with serum cortisol and cortisone. However, salivary cortisone is not affected by ingestion of hydrocortisone, such that it might be a superior surrogate marker of serum cortisol than salivary cortisol. ${ }^{28}$ In another study of adrenal insufficiency, measurement of salivary cortisol level was found to reduce corticotropin stimulation test by $34 \%$, with a minimal salivary cortisol concentration of $1.0 \mathrm{nmol} / \mathrm{L}$ and a maximum concentration of $5.9 \mathrm{nmol} / \mathrm{L} .{ }^{29} \mathrm{In}$ the present study, salivary cortisol and cortisone were measured in patients with CU. We found them also to be negatively correlated with stress severity, and salivary cortisone was positively 
correlated with urticaria control status. Furthermore, the K-UCT was found to be a significant predictor of salivary cortisone levels through structural equation modeling. Therefore, since urticaria control status contributes directly to increasing stress levels, salivary cortisone may be a useful parameter with which to easily index chronic stress in patients with CU.

There are some limitations to the questionnaire itself. First, the K-UCT only reflects a subject's feelings. Secondly, there are still unnatural Korean expressions in the questionnaire, which can be difficult to answer in short time. Further studies should seek to address these limitations in order to better reflect the control status of CU and to make answering the questionnaire easier.

In conclusion, the present study demonstrated that the Korean version of the UCT is a useful tool for evaluating urticaria control status in Korean patients with CU. Our results also indicate that the original German UCT is a well-organized tool to be widely accepted in other countries with cultural and linguistic divergence. In addition, we found salivary cortisone levels to be correlated with the K-UCT, supporting the implication of chronic stress in the pathogenesis of CU.

\section{ACKNOWLEDGMENTS}

Original German version of the Urticaria Control Test was provided from MOXIE (Berlin, Germany). We thank Dr. Karsten Weller (Charite - Universitätsmedizin Berlin) who helped backward translation of the Korean UCT into German. This study was supported partly by a grant from Investigator-Initiated Studies Program of Novartis Korea and partly by a grant of the Korea Health Technology R\&D Project through the Korea Health Industry Development Institute (KHIDI), funded by the Ministry of Health \& Welfare, Republic of Korea (HI16C0992).

\section{SUPPLEMENTARY MATERIAL}

\section{Supplementary Fig. S1}

The Korean version of the Urticaria Control Test (K-UCT).

Click here to view

\section{REFERENCES}

1. Zuberbier T, Aberer W, Asero R, Bindslev-Jensen C, Brzoza Z, Canonica GW, et al. The EAACI/GA²LEN/ EDF/WAO Guideline for the definition, classification, diagnosis, and management of urticaria: the 2013 revision and update. Allergy 2014;69:868-87. PUBMED | CROSSREF

2. O'Donnell BF, Lawlor F, Simpson J, Morgan M, Greaves MW. The impact of chronic urticaria on the quality of life. Br J Dermatol 1997;136:197-201. PUBMED | CROSSREF

3. Zuberbier T, Balke M, Worm M, Edenharter G, Maurer M. Epidemiology of urticaria: a representative cross-sectional population survey. Clin Exp Dermatol 2010;35:869-73. PUBMED | CROSSREF 
4. Lee N, Lee JD, Lee HY, Kang DR, Ye YM. Epidemiology of chronic urticaria in Korea using the Korean health insurance database, 2010-2014. Allergy Asthma Immunol Res 2017;9:438-45. PUBMED | CROSSREF

5. Weller K, Groffik A, Church MK, Hawro T, Krause K, Metz M, et al. Development and validation of the Urticaria Control Test: a patient-reported outcome instrument for assessing urticaria control. J Allergy Clin Immunol 2014;133:1365-72, 1372.e1-6. PUBMED | CROSSREF

6. Yang HY, Sun CC, Wu YC, Wang JD. Stress, insomnia, and chronic idiopathic urticaria--a case-control study. J Formos Med Assoc 2005.104:254-63. PUBMED

7. Dyke SM, Carey BS, Kaminski ER. Effect of stress on basophil function in chronic idiopathic urticaria. Clin Exp Allergy 2008.38:86-92. PUBMED

8. Hellhammer DH, Wüst S, Kudielka BM. Salivary cortisol as a biomarker in stress research. Psychoneuroendocrinology 2009;34:163-71. PUBMED | CROSSREF

9. Smith RE, Maguire JA, Stein-Oakley AN, Sasano H, Takahashi K, Fukushima K, et al. Localization of 11 beta-hydroxysteroid dehydrogenase type II in human epithelial tissues. J Clin Endocrinol Metab 1996;81:3244-8. PUBMED

10. Bae YJ, Gaudl A, Jaeger S, Stadelmann S, Hiemisch A, Kiess W, et al. Immunoassay or LC-MS/MS for the measurement of salivary cortisol in children? Clin Chem Lab Med 2016;54:811-22. PUBMED | CROSSREF

11. Blair J, Adaway J, Keevil B, Ross R. Salivary cortisol and cortisone in the clinical setting. Curr Opin Endocrinol Diabetes Obes 2017;24:161-8. PUBMED | CROSSREF

12. Konstantinou GN, Asero R, Maurer M, Sabroe RA, Schmid-Grendelmeier P, Grattan CE. EAACI/GA²LEN task force consensus report: the autologous serum skin test in urticaria. Allergy 2009;64:1256-68. PUBMED | CROSSREF

13. Hawro T, Ohanyan T, Schoepke N, Metz M, Peveling-Oberhag A, Staubach P, et al. Comparison and interpretability of the available Urticaria Activity Scores. Allergy 2018;73:251-5. PUBMED | CROSSREF

14. Ye YM, Park JW, Kim SH, Choi JH, Hur GY, Lee HY, et al. Clinical evaluation of the computerized chronic urticaria-specific quality of life questionnaire in Korean patients with chronic urticaria. Clin Exp Dermatol 2012;37:722-8. PUBMED | CROSSREF

15. Lee EH, Chung BY, Suh CH, Jung JY. Korean versions of the Perceived Stress Scale (PSS-14, 10 and 4): psychometric evaluation in patients with chronic disease. Scand J Caring Sci 2015;29:183-92. PUBMED | CROSSREF

16. Barth J, de Boer WE, Busse JW, Hoving JL, Kedzia S, Couban R, et al. Inter-rater agreement in evaluation of disability: systematic review of reproducibility studies. BMJ 2017;356:j14. PUBMED | CROSSREF

17. Gaudl A, Kratzsch J, Bae YJ, Kiess W, Thiery J, Ceglarek U. Liquid chromatography quadrupole linear ion trap mass spectrometry for quantitative steroid hormone analysis in plasma, urine, saliva and hair. J Chromatogr A 2016;1464:64-71. PUBMED | CROSSREF

18. Soh SE, McGinley JL, Watts JJ, Iansek R, Murphy AT, Menz HB, et al. Determinants of health-related quality of life in people with Parkinson's disease: a path analysis. Qual Life Res 2013;22:1543-53. PUBMED | CROSSREF

19. Trinh HK, Pham DL, Ban GY, Lee HY, Park HS, Ye YM. Altered systemic adipokines in patients with chronic urticaria. Int Arch Allergy Immunol 2016;171:102-10. PUBMED | CROSSREF

20. Baiardini I, Braido F, Bindslev-Jensen C, Bousquet PJ, Brzoza Z, Canonica GW, et al. Recommendations for assessing patient-reported outcomes and health-related quality of life in patients with urticaria: a GA²LEN taskforce position paper. Allergy 2011;66:840-4. PUBMED | CROSSREF

21. Kulthanan K, Chularojanamontri L, Tuchinda P, Rujitharanawong C, Maurer M, Weller K. Validity, reliability and interpretability of the Thai version of the Urticaria Control Test (UCT). Health Qual Life Outcomes 2016;14:61. PUBMED | CROSSREF 
22. García-Díez I, Curto-Barredo L, Weller K, Pujol RM, Maurer M, Giménez-Arnau AM. Cross-cultural adaptation of the Urticaria Control Test from German to Castilian Spanish. Actas Dermosifiliogr 2015;106:746-52. PUBMED | CROSSREF

23. Ohanyan T, Schoepke N, Bolukbasi B, Metz M, Hawro T, Zuberbier T, et al. Responsiveness and minimal important difference of the Urticaria Control Test. J Allergy Clin Immunol 2017;140:1710-1713.e11. PUBMED | CROSSREF

24. Lindsay K, Goulding J, Solomon M, Broom B. Treating chronic spontaneous urticaria using a brief 'whole person' treatment approach: a proof-of-concept study. Clin Transl Allergy 2015;5:40. PUBMED | CROSSREF

25. Hammen C, Kim EY, Eberhart NK, Brennan PA. Chronic and acute stress and the prediction of major depression in women. Depress Anxiety 2009;26:718-23.

PUBMED | CROSSREF

26. Varghese R, Rajappa M, Chandrashekar L, Kattimani S, Archana M, Munisamy M, et al. Association among stress, hypocortisolism, systemic inflammation, and disease severity in chronic urticaria. Ann Allergy Asthma Immunol 2016;116:344-348.e1. PUBMED | CROSSREF

27. Kim JE, Cho BK, Cho DH, Park HJ. Expression of hypothalamic-pituitary-adrenal axis in common skin diseases: evidence of its association with stress-related disease activity. Acta Derm Venereol 2013;93:387-93. PUBMED | CROSSREF

28. Debono M, Harrison RF, Whitaker MJ, Eckland D, Arlt W, Keevil BG, et al. Salivary cortisone reflects cortisol exposure under physiological conditions and after hydrocortisone. J Clin Endocrinol Metab 2016;101:1469-77. PUBMED | CROSSREF

29. Langelaan ML, Kisters JM, Oosterwerff MM, Boer AK. Salivary cortisol in the diagnosis of adrenal insufficiency: cost efficient and patient friendly. Endocr Connect 2018;7:560-6. PUBMED | CROSSREF 